\title{
Subgingival dysbiosis in smoker and non-smoker patients with chronic periodontitis
}

\author{
LORENA CORETTI ${ }^{1,2}$, MARIELLA CUOMO ${ }^{1,2}$, ERMANNO FLORIO ${ }^{1,2}$, DOMENICO PALUMBO $^{2}$, \\ SIMONA KELLER ${ }^{1,2}$, RAFFAELA PERO $^{2}$, LORENZO CHIARIOTTI $^{1,2}$, \\ FRANCESCA LEMBO ${ }^{3}$ and CARLO CAFIERO ${ }^{4}$ \\ ${ }^{1}$ Institute of Experimental Endocrinology and Oncology, National Research Council; \\ Departments of ${ }^{2}$ Molecular Medicine and Medical Biotechnology; ${ }^{3}$ Pharmacy and ${ }^{4}$ Neuroscience, \\ Reproductive Sciences and Dentistry, University of Naples Federico II, I-80131 Naples, Italy
}

Received August 31, 2016; Accepted December 12, 2016

DOI: $10.3892 / \mathrm{mmr} .2017 .6269$

\begin{abstract}
Periodontitis is one of the most common oral inflammatory diseases, and results in connective tissue degradation and gradual tooth loss. It manifests with formation of periodontal pockets, in which anaerobic and Gram-negative bacteria proliferate rapidly. Consequently, alteration of the subgingival microbiota is considered the primary etiologic agent of periodontitis. Previous studies have reported that smokers are at increased risk of periodontal disease, in both prevalence and severity, indicating that smoking is a risk factor for the onset and progression of the pathology. In the present study, 16S rRNA sequencing was employed to assess the subgingival microbiota in 6 smoker patients with chronic periodontitis, 6 non-smoker patients with chronic periodontitis and 8 healthy controls. The results demonstrated significant alterations in the microbial structure of periodontitis patients. High relative abundance of Parvimonans, Desulfubulbus, Paludiba cter, Haemophilus, and Sphaerochaeta genera characterized subgingival microbiota of periodontitis patients, both smokers and non-smokers. Due to the high precision and sensitivity of the 16S rRNA sequencing method, analysis for low-abundant genera (including Pedobacter, Granulicatella, Paracoccus, Atopobium, Bifidobacterium, Coprococcus, Oridobacteriu, Peptococcus, Oscillospira and Akkermansia) was feasible,
\end{abstract}

Correspondence to: Dr Francesca Lembo, Department of Pharmacy, University of Naples Federico II, 49 Via D. Montesano, I-80131 Naples, Italy

E-mail: frlembo@unina.it

Dr Lorenzo Chiariotti, Institute of Experimental Endocrinology and Oncology, National Research Council, 5 Via S. Pansini, I-80131 Naples, Italy

E-mail: chiariot@unina.it

Key words: subgingival microbiota, $16 \mathrm{~S}$ rRNA gene next generation sequencing, chronic periodontitis, smoker and non-smoker subgingival microbiota profiles, key phylotypes of chronic periodontitis and revealed novel phylotypes associated with periodontitis. Of note, a major microbial community alteration was evident in smoker patients, suggesting an association between smoking and severity of subgingival dysbiosis. The present study confirmed that chronic periodontitis is a polymicrobial disease where changes in the equilibrium of subgingival microbiota contribute to severity of pathology.

\section{Introduction}

The oral cavity is an ideal environment for microbial growth, with the oral microbiota comprising of a large variety of species at high densities. It is well established that an equilibrated microbiota contributes to a healthy status for the host (1). Conversely, changes in the balance between different bacterial species that inhabit the human body contribute to the pathogenesis of several diseases (2). An alteration in the composition of subgingival microbiota is one of the most common causes of periodontal disease (3). Periodontitis is a microbial infection that manifests with inflammation of gingival tissue (4). Bacteria, especially Gram-negative species, invade the supporting structures of teeth, forming subgingival oral biofilms in which pro-inflammatory molecules are released. The affected tissues (bone, gingiva and periodontal ligament) suffer damage, the extent of which depends on the different immune responses of each individual (5), indicating that periodontitis may manifest concurrently with other systemic diseases (6). Based on the severity of the phenotype, two forms of periodontitis are identified: Chronic periodontitis that affects up of $50 \%$ of the global population with a slow rate of progression, and aggressive periodontitis with a rapid loss of attachment and bone destruction (7). Although the oral microbiota is different in each patient, several studies have demonstrated that three principal bacteria are frequently identified in the dental plaque of patients with periodontitis, classified as the 'red complex' organisms: Tannerella forsythia, Porphyromonas gingivalis, and Treponema denticola (8). These Gram-negative bacteria are involved in the etiopathogenesis of periodontitis, most likely due to their production of various virulence factors, including collagenase, protease and endotoxin. Although subgingival microbiota are important in the development of periodontitis, 
other factors also contribute to the development and severity of the disease, such as smoking (9). The harmful components of smoke can alter the oral bacterial composition, influencing the level of oxygenation and, consequently, the growth of beneficial aerobic bacteria (10). Furthermore, smoking can modify the host immune response making the oral cavity more susceptible to proliferation of pathogenic bacterial species (11). Not surprisingly, the red complex organisms associated with periodontitis are anaerobic bacteria that can proliferate more easily in an environment worsened by smoke. Despite previous studies demonstrating that the onset and the progression of periodontitis may be compounded by smoking (12), analysis of the microbiota based on culture techniques has revealed conflicting results $(13,14)$. Only 200 bacterial species that inhabit the oral cavity have been cultured, however $\sim 600$ species have been identified by $16 \mathrm{~S}$ rDNA sequencing (15). In the present study, a metagenomic analysis using high coverage next generation sequencing (NGS) was performed, in order to define the microbiota composition with high precision. The microbial composition from gingival tissues was analyzed in order to identify both strongly adhered and biofilm forming bacteria. The present study demonstrated a general alteration in microbial composition in subgingival tissues in patients with chronic periodontitis. Key phylotypes characterized the subgingival microbiota of chronic periodontitis patients compared with healthy ones. Finally, data analysis revealed a potential contribution of smoking in the alteration of microbial equilibrium in subgingival tissues, thus worsening the severity of periodontal disease.

\section{Materials and methods}

Selection of patient study group. Twenty patients were recruited among consecutive patients referred to the Periodontal Surgery and Osteo-integrated Implantology Clinical Unit at Department of Neuroscience, Reproductive Sciences and Dentistry of the University of Naples Federico II (Naples, Italy). Patients were included in the study if they fulfilled the following criteria: Good general health, no previous periodontal treatment in the last 2 years, no use of antibiotics that could affect the subgingival microbiota in the past 6 months, no pregnancy and presence of at least 20 natural teeth. The selected patients were then divided into three groups: i) 8 non-smoker, periodontally healthy patients affected by dysodontiasis of mandibular third molar [3 males and 5 females, acting as controls (CTRLs)], ii) 6 non-smoker patients affected by chronic moderate to severe periodontitis (1 male and 5 females; termed here PCnoS), and iii) 6 smokers affected by chronic moderate to severe periodontitis ( 2 males and 4 females; termed here PCS). The severity of periodontitis was categorized on the basis of the amount of clinical attachment loss, as follows: Slight, 1-2 mm; moderate, 3-4 mm; severe, $\geq 5 \mathrm{~mm}$ (7). A patient was defined as a smoker if he/she was currently smoking and had been smoking for at least 10 years and as a non-smoker if he/she had never smoked or quit smoking longer than 10 years prior to recruitment for the present study. Patients who agreed to participate in the study signed a written informed consent. The protocol of the study conformed to ethical regulations by the Department of Neuroscience, Reproductive Sciences and Dentistry,
University of Naples Federico II. At the baseline appointment, the following clinical measurements were recorded: Plaque (presence/absence), bleeding on probing, probing pocket depth and clinical attachment loss.

Subgingival samples collection. Eight periodontally healthy patients aged 21-63 years who required removal of impacted mandibular third molars were included in this study. No acute infection of the soft tissues covering the impacted tooth was observed. Following administration of locoregional anesthesia, a full-thickness mucoperiosteal flap was raised for the extraction of the third molar. The flap incision extended from the vestibular side of the retromolar trigon to the second molar, corresponding to its distolingual cuspid. The incision continued vestibularly around the intrasulcular surface of the second molar, then an additional cut was made distally to the papilla between the first and second molars, on a $45^{\circ}$ angle, which extended vestibularly for 2 to $3 \mathrm{~cm}$, as described by Sammartino et al (16). A small quantity of soft tissue was picked up from the surgical site and stored in a $1.5 \mathrm{ml}$ microcentrifuge tube and frozen at $-80^{\circ} \mathrm{C}$ for further processing. Subsequently, an osteotomy was performed using a Lindemann bur under constant irrigation, followed by an odontotomy using a diamond bur. Following the extraction of the included third molar, the root surface of the second molar was scaled. The flap was sutured by the use of 2-0 silk sutures. Twelve patients aged 42-68 for the PCnoS group and aged 39-55 for the PCS group, affected by severe to moderate chronic periodontitis were recruited for surgery. Modified Widman Flap was performed (17). Following administration of locoregional anesthesia, intrasulcular and interdental incisions were made. A flap was raised and reflected by the use of an elevator. Granulation tissue was isolated, removed and stored in a $1.5 \mathrm{ml}$ microcentrifuge tube and frozen at $-80^{\circ} \mathrm{C}$ for further processing. Scaling and root planning of the exposed root surfaces were accurately made and finally the flap was repositioned and sutured using 4-0 silk sutures.

DNA extraction and 16S metagenomic sequencing. Bacterial genomic DNA was extracted from liquid nitrogen-pulverized tissue samples using the DNeasy Blood \& Tissue kit (Qiagen Gmbh, Hilden, Germany) according to manufacturer's instructions. The quantity and quality of DNA was determined by spectrophotometric measurements (NanoDrop; Thermo Fisher Scientific Inc., Waltham, MA, USA), and all DNA samples were stored at $-20^{\circ} \mathrm{C}$ until further processing for sequencing.

The V3-V4 region of the 16S ribosomal RNA (rRNA) gene from each DNA sample was amplified and prepared for sequencing according to the protocol $16 \mathrm{~S}$ Metagenomic Sequencing Library Preparation for Illumina Miseq System (18). This region of the rRNA gene has previously been described as an optimal marker for bacterial taxonomic classification (19).

Barcoded amplicons were mixed in equal amounts based on concentrations determined by Qubit Fluorometer (Invitrogen; Thermo Fisher Scientific, Inc.) and library sizes were assessed using a Bioanalyzer DNA 1000 chip (Agilent Technologies Gmbh, Waldbronn, Germany). Normalized libraries (4 nM) were pooled, denatured with $\mathrm{NaOH}(0.2 \mathrm{~N})$ for $5 \mathrm{~min}$ at room temperature, diluted to $10 \mathrm{pM}$ with HT1 buffer (provided by 
the Illumina MiSeq v3 Reagent kit; Illumina, Inc., San Diego, CA, USA) and combined with $25 \%$ (v/v) denatured $10 \mathrm{pM}$ PhiX (Illumina, Inc.), according to Illumina guidelines (20). Sequencing run was performed on the Illumina MiSeq system using v3 reagents for $2 \times 281$ cycles (Illumina, Inc.).

Metagenomic data analysis. V3-V4 16S rDNA FASTQ paired-end reads were pre-processed with PEAR (21) in order to assemble reads with an overlap of at least 40 nucleotides, and to remove low quality sequences (PHRED score $\leq 33$ ) that were shorter than $400 \mathrm{bp}$ and longer than $500 \mathrm{bp}$. Passing filter sequences were then processed with PRINSEQ (22) in order to obtain FASTA and quality files for further analyses. Pick of operational taxonomic units (OTUs), taxonomic assignment and diversity analyses were conducted using Quantitative Insights Into Microbial Ecology (QIIME, version 1.8.0) (23). High-quality sequences were used to pick OTUs at $97 \%$ sequence similarity from Greengenes $16 \mathrm{~S}$ gene database (GG; May 2013 version) (24) with a closed reference-based OTU picking method. The GG database was used to taxonomically classify the identified OTUs and to compute their distribution across different taxonomic levels. To avoid sample size biases in subsequent analyses, samples were normalized to 34,559 sequences/sample using a sequence rarefaction procedure. Species heterogeneity in each sample was assessed employing two Alpha diversity metrics (the number Observed species and the Shannon entropy) and compared using one-way analysis of variance (ANOVA) followed by Tukey's multiple comparison post-hoc test. Unweighted Unifrac distances were calculated to analyze OTUs diversity among sample communities (beta diversity). Nonparametric multivariate analysis of changes in community structure was performed using the analysis of similarities (ANOSIM) method (25) with 999 permutations, to test the force of microbial communities grouping. Statistical differences in OTU frequencies among groups across different taxonomic levels were assessed with linear discriminative analysis (LDA) effect size analysis (LEfSe, logarithmic LDA score $>2$ and $\mathrm{P}<0.05$ for Kruskal-Wallis test) (26). The normalized OTU table, corrected for multiple 16S rRNA gene copy number, was used for metagenome prediction in PICRUSt (27). Kyoto encyclopedia of genes and genomes (KEGG) (28) ortholog abundances were categorized by function at hierarchical pathway level 3 and compared among groups with LEfSe (LDA score $>2$ and $\mathrm{P}<0.05$ ).

Data Deposition. The sequences reported in this study are deposited on the 'European Nucleotide Archive' server (www. ebi.ac.uk/ena) under the accession number PRJEB17957.

\section{Results}

Subgingival microbial diversity associated with chronic periodontitis. The subgingival microbiota of patients with chronic periodontitis and healthy controls (CTRLs) was analyzed. Among periodontitis patients, the samples were classified into 2 groups: Samples from smoker patients with chronic periodontitis (PCS) and samples from non-smoker patients with chronic periodontitis (PCnoS). The different subgingival microbiota compositions in 8 CTRLs, 6 PCS and 6 PCnoS patients were studied by high-throughput sequencing of the
16S rDNA V3-V4 hypervariable region on an Illumina MiSeq platform. This region is commonly amplified in $16 \mathrm{~S}$ rRNA gene-based metagenomic studies, as it has been determined to provide optimal bacterial taxonomic coverage and taxonomic resolution $(19,29)$. High quality reads $(>2,000,000)$, with an average of 110,169 sequences/sample, were assigned into 3,891 OTUs; following the rarefaction procedure, 34,559 rarefied sequences/sample were considered, representing 2,937 OTUs, in order to describe the differences in subgingival bacterial communities between samples. The alpha and beta diversity among the three groups were measured in order to evaluate the bacterial diversity in terms of species richness and community diversity. Alpha diversity results revealed that the subgingival bacterial population of PCS and PCnoS groups exhibited a significant decrease of species richness, demonstrated by number of OTUs $(\mathrm{P}<0.05$; Fig. 1A) and of microbial diversity (Shannon index; $\mathrm{P}<0.05$; Fig. 1B), compared with CTRLs. Beta diversity, representing the distances among samples and groups in terms of bacterial community composition, was then measured. Using the ANOSIM R statistic, an index based on rank dissimilarity, dissimilarity between groups based on Unweighted Unifract distances was measured. ANOSIM analysis revealed significant differences in subgingival bacterial assortment between PCS and CTRL groups (ANOSIM $\mathrm{R}=0.21, \mathrm{P}=0.03$ ), while no significant differences in bacterial assortment were identified between PCnoS and the other groups. In fact, a Principal Coordinate Analysis (PCoA) plot displayed clustering of smoker patients away from healthy controls, while non-smoker samples clustered closely to healthy controls (Fig. 1C).

Description of subgingival microbiota in PCS and PCnoS patients compared with CTRL: identification of key phylotypes. Sequencing analysis revealed that Actinobacteria, Bacteroidetes, Firmicutes, Fusobacteria and Proteobacteria were the dominant phyla of subgingival microbiota in all groups (Fig. 2A). Amongst the 22 bacterial phyla identified, comparison of relative abundances by LEfSe analysis revealed no significant differences between PCnoS and CTRL (Fig. 2A). By contrast, significantly higher levels of Fusobacteria and lower levels of Cyanobacteria, Firmicutes, SR1 (also termed Absconditabacteria) (30) and Verrucomicrobia phyla distinguished PCS and CTRL bacterial communities $(\mathrm{P}<0.05$; Fig. 2A). At the genus level, periodontitis patients were characterized by alterations in relative abundance of several bacterial genera compared to the CTRL group. According to alpha diversity results, the microbial communities of CTRL and PCnoS groups were not dominated by a single genus, while the PCS group showed a predominance in the Fusobacterium genus (Fig. 2B). In fact, Fusobacterium was more abundant in the PCS group (32.84\% of total microbiota) compared with CTRL and PCnoS groups (13.78 and 14.96\%, respectively; Fig. 2B), even though these effects were not statistically significant.

Key phylotypes were identified from direct comparisons between the patient groups (PCS/CTRL, PCnoS/CTRL and $\mathrm{PCS} / \mathrm{PCnoS}$ ) using LEfSe algorithm analysis. Among the 346 genera detected across all samples, 56 genera differentiated between the CTRL and PCS groups. A distinct and smaller set of bacterial genera (31 genera) differentiated the PCnoS 

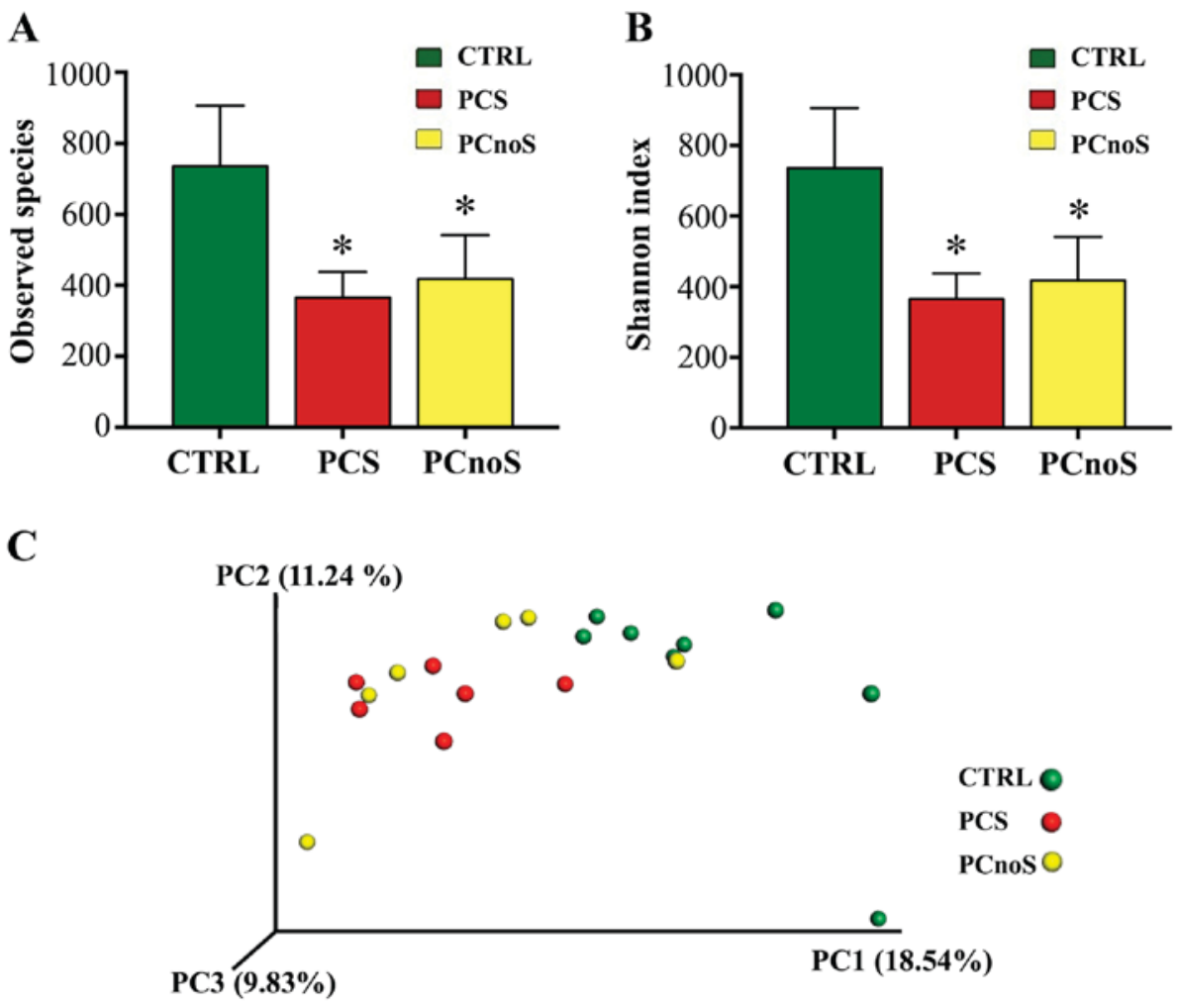

Figure 1. Comparison of subgingival microbial diversity among PCS, PCnoS and CTRL groups. Diversity within bacterial communities is demonstrated by (A) the number of observed species and (B) the Shannon Index. Results are presented as the mean \pm standard deviation. *P<0.05 vs. CTRL, by Tukey's test for multiple comparisons. (C) Diversity among bacterial communities is displayed in the principal coordinate analysis plot, based on unweighted Unifrac distances $(34,559$ sequences/sample), with the amount of variance along each axis in brackets. CTRL, healthy controls; PCS, smokers with periodontitis; PCnoS, non-smokers with periodontitis.

and CTRLs groups, while only 10 different genera were evident between the PCS and PCnoS groups (Fig. 2C). Table I describes the relative abundances of key phylotypes identified by LEfSe analysis as significantly different between groups. The dysbiotic profile of PCS subgingival microbiota compared with CTRL was characterized by significant increase of Desulfobulbus, Sphaerochaeta, and unclassified genera of Mogibacteriaceaea and Tissierellaceae, and significant decrease of Pedobacter, Granulicatella, Paracoccus, Gemellaceae, Clostridiaceae, Ruminococcaceae, Sphingomonadaceae, Pseudomonadaceae, Xanthomondaceae, and an unclassified genus of S24-7 (Table I). Notably, Pseudoramibacter was significantly more abundant in both PCS and PCnoS compared with CTRL (Table I). Finally, Bacteroides, Prev otella, Veillonella, Pseudomonas, and unclassified genera of Enterobacteriaceae, Rikenellaceae, Lachnospiraceae and Clostridiales were significantly less abundant or even absent in the PCS and PCnoS groups compared with the CTRL group (Table I).

Predictive subgingival microbiota functions in chronic periodontitis patients. In order to evaluate putative biological functions of PCS, PCnoS and CTRL subgingival microbial communities, a PICRUSt analysis was performed. Based on bacterial sequences, the comparison of microbiota-related gene functions between periodontitis and healthy samples demonstrated alterations in 46 metabolic pathways in PCS and in 8 pathways in $\mathrm{PCnoS}$ subgingival microbiota compared with CTRL (Fig 3). Among the altered metabolic functions in the PCS group compared with CTRL, an enrichment of genes encoding peptidases, enzymes for lipopolysaccharide biosynthesis, and proteins involved in cell motility and secretions was evident (Fig. 3). Conversely, enzymes for biosynthesis and biodegradation of secondary metabolites, fatty acid metabolism and amino acid biosynthesis were highly represented in healthy CTRL subgingival microbiotacompared with the PCS group (Fig. 3). In addition, enzymes involved in cell motility and secretion, homologous recombination, peptidase and DNA replication were more heavily represented in the subgingival microbiota of PCS compared with PCnoS (Fig. 3).

\section{Discussion}

The bacterial composition of healthy and periodontal gingival sulcus has largely been described $(31,32)$. The present study expanded on previous published information about periodontal subgingival microbiota, by next generation sequencing on an Illumina Miseq platform. This approach allows identification of a larger range of bacterial sequences, increasing the sensitivity of the analysis. In order to avoid loss of bacterial taxa that are able to adhere to subgingival oral biofilms and aggravate periodontitis pathology, bacterial DNA was extracted directly from gingival tissue. Furthermore, the involvement of smoking as an environmental factor and its role in altering the microbiota composition of periodontitis patients was assessed. The present study demonstrated that alpha diversity decreased in both smoker (PCS) and non-smoker (PCnoS) periodontitis patients compared with healthy controls. The lower bacterial 
$\mathbf{A}$

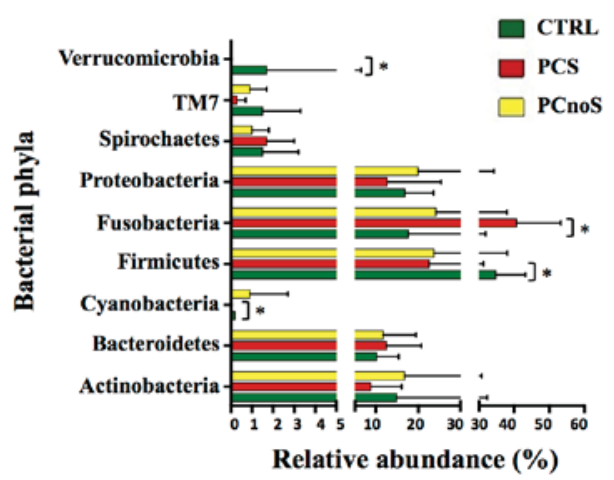

B
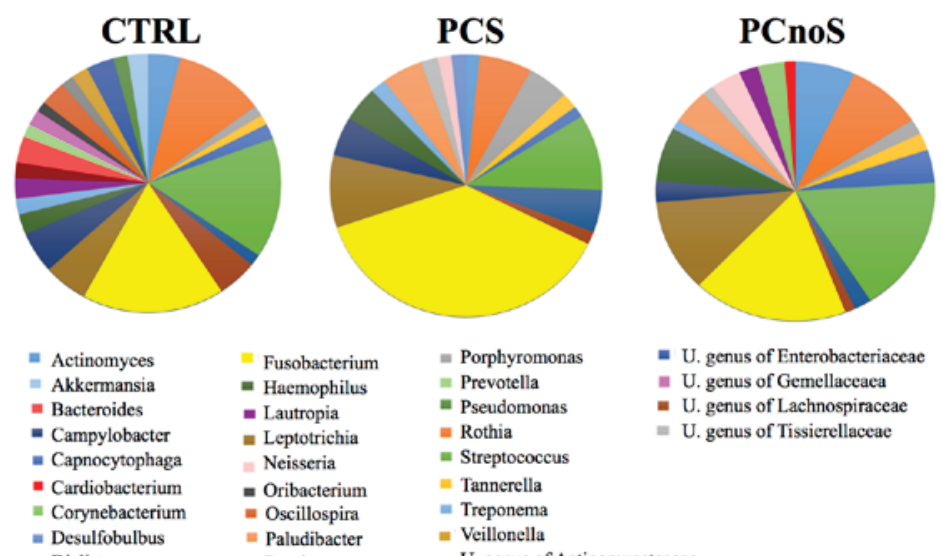

= Fusobacterium

= Porphyromonas

$=$ Prevotella

- Lautropia

= Pseudomonas

- Streptococ

- Leptotrichia

Streptococc

Neisseria

Oribacterium

- Oscillospira
= Paludibacter

$=$ Tannerella

$=$ Treponem

$=$ Veillonella

= Dialister $\quad$ = Parvimonas

- U. genus of Actinomycetaceac

C

346 bacterial genera detected across all samples

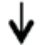

LEfSe algorithm analysis to identified key phylotypes
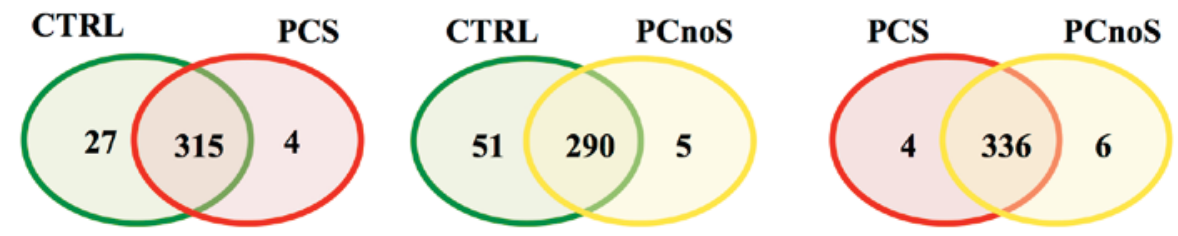

Figure 2. Subgingival microbiota composition in PCS, PCnoS and CTRL groups. (A) The percentage distribution of bacterial phyla identified with relative abundance $>1 \%$. The mean \pm standard error of the mean are plotted. Significant differences are indicated by ${ }^{*} \mathrm{P}<0.05$. (B) Pie charts representing dominant genera with relative abundance $>1 \%$ of the total reads. The mean values of the major genera are presented for each study group. (C) Number of key and shared phylotypes in CTRLvsPCS, CTRLvsPCnoS and PCSvsPCnoS comparisons as determined by LEfSe algorithm analysis (LDA $>3$ with alpha <0.05). PCS, smokers with periodontitis; PCnoS, non-smokers with periodontitis; CTRL, healthy controls; U, unknown.

richness of periodontitis samples was confirmed by taxonomic classification of the majority $(41 \%)$ of total reads in Gram-negative and anaerobic Fusobacteria phylum, specifically in the PCS group. In addition, beta diversity analysis demonstrated a different composition in subgingival microbiota between PCS and CTRLs. The PCoA plot revealed a separate clustering of the two groups, indicating a diversity in their bacterial communities.

In the present study, the potential pathogenic role of bacteria identified in high levels in subgingival microbiota of PCS and PCnoS groups was also explored. Genera including Parvimonans, Desulfubulbus, Paludibacter, Hae mophilus, and Sphaerochaeta were discovered to be associated with periodontitis. Although Parvimonans normally inhabits the oral cavity, it has been isolated in high numbers in periodontitis patients and is associated with destruction of periodontal tissue (33). Desulfubulbus and Paludibacter have also previously been associated with periodontitis (34) and high concentrations have been linked to worse periodontal therapeutic response (35). In the present study, identification of Desulfobulbus and Paludibacter was increased in PCS samples compared with PCnoS and CTRLs, corroborating the hypothesis that smoking interferes with microbiota composition during periodontal disease. Sphaerochaeta has been previously linked with a large number of pathologies, such as syphilis and Lyme disease (36), but no association to periodontitis had been previously reported. Similarly, the presence of Haemophilus in subgingival microbiota of periodontal patients had not been demonstrated to date. Because Haemophilus grows well in blood-enriched culture media (37), its presence may be explained by the bleeding gums typically featured in periodontal disease. Periodontitis-associated dysbiosis was also 
Table I. Key genera among PCS, PCnoS and CTRL groups.

\begin{tabular}{|c|c|c|c|c|}
\hline Phyla & Genera & $\%$ in CTRL & $\%$ in PCS & $\%$ in $\mathrm{PCnoS}$ \\
\hline \multirow[t]{3}{*}{ Actinobacteria } & Atopobium & $0.19 \pm 0.05$ & $0.57 \pm 0.37^{\mathrm{c}}$ & $0.57 \pm 0.37$ \\
\hline & Bifidobacterium & $0.25 \pm 0.10$ & $0.12 \pm 0.07$ & $0.02 \pm 0.02^{\mathrm{b}}$ \\
\hline & U. Actinomycetales & $0.06 \pm 0.06$ & $0^{\mathrm{c}}$ & $0.01 \pm 0.00$ \\
\hline \multirow[t]{5}{*}{ Bacteriodetes } & Bacteroides & $2.52 \pm 2.21$ & $0.01 \pm 0.01^{\mathrm{a}}$ & $0.03 \pm 0.02^{\mathrm{b}}$ \\
\hline & Pedobacter & $0.003 \pm 0.00$ & $0^{\mathrm{a}}$ & $0.001 \pm 0.00$ \\
\hline & Prevotella & $1.46 \pm 0.27$ & $0.40 \pm 0.14^{\mathrm{a}}$ & $0.26 \pm 0.09^{b}$ \\
\hline & U. Rikenellaceae & $0.80 \pm 0.50$ & $0.01 \pm 0.00^{\mathrm{a}}$ & $0.06 \pm 0.06^{\mathrm{b}}$ \\
\hline & U.S24-7 & $0.30 \pm 0.22$ & $0.03 \pm 0.02^{\mathrm{a}}$ & $0.04 \pm 0.04$ \\
\hline \multirow[t]{14}{*}{ Firmicutes } & Faecalibacterium & $0.14 \pm 0.09$ & 0 & $0^{\mathrm{b}}$ \\
\hline & Granulicatella & $0.78 \pm 0.32$ & $0.11 \pm 0.07^{\mathrm{a}}$ & $0.22 \pm 0.02$ \\
\hline & Oribacterium & $1.01 \pm 0.36$ & $0.92 \pm 0.52$ & $0.19 \pm 0.00^{\mathrm{b}}$ \\
\hline & Oscillospira & $2.33 \pm 1.41$ & $0.02 \pm 0.01$ & $0.12 \pm 0.02^{b}$ \\
\hline & Pseudoramibacter & $0.01 \pm 0.01$ & $0.02 \pm 0.01$ & $0.24 \pm 0.01^{b}$ \\
\hline & Ruminococcus & $0.19 \pm 0.11$ & $0.001 \pm 0.00$ & $0^{\mathrm{b}}$ \\
\hline & Veillonella & $1.68 \pm 0.39$ & $0.27 \pm 0.12^{\mathrm{a}}$ & $0.23 \pm 0.04^{\mathrm{b}}$ \\
\hline & U. Clostridiaceae & $0.67 \pm 0.50$ & $0.04 \pm 0.04^{\mathrm{a}}$ & $0.09 \pm 0.07$ \\
\hline & U. Clostridiales & $0.07 \pm 0.03$ & $0.17 \pm 0.05^{\mathrm{a}}$ & $0.50 \pm 0.07^{\mathrm{b}}$ \\
\hline & U. Gemellaceae & $1.53 \pm 0.41$ & $0.07 \pm 0.04^{\mathrm{a}}$ & $0.43 \pm 0.04$ \\
\hline & U. Lachnospiraceae & $4.21 \pm 0.79$ & $1.42 \pm 0.83$ & $1.02 \pm 1.07^{\mathrm{b}}$ \\
\hline & U. Mogibacteriaceae & $0.68 \pm 0.29$ & $1.83 \pm 0.30^{\mathrm{a}}$ & $0.79 \pm 1.05$ \\
\hline & U. Ruminococcaceae & $1.02 \pm 0.64$ & $0.02 \pm 0.01^{\mathrm{a}}$ & $0.07 \pm 0.06$ \\
\hline & U. Tissierellaceae & $0.08 \pm 0.07$ & $1.78 \pm 1.27^{\mathrm{a}}$ & $1.07 \pm 1.08$ \\
\hline Fusobacteria & U. Leptotrichiaceae & $0.34 \pm 0.34$ & $0.33 \pm 0.21^{\mathrm{c}}$ & 0 \\
\hline \multirow[t]{10}{*}{ Proteobacteria } & Cardiobacterium & $0.48 \pm 0.17$ & $0.18 \pm 0.12^{c}$ & $1 \pm 0.01$ \\
\hline & Desulfobulbus & $0.05 \pm 0.02$ & $1.49 \pm 0.64^{\mathrm{a}, \mathrm{c}}$ & $0.14 \pm 1.02$ \\
\hline & Lautropia & $1.85 \pm 1.55$ & $0.61 \pm 0.51^{\mathrm{c}}$ & $1.85 \pm 0.07$ \\
\hline & Ochrobactrum & $0.01 \pm 0.01$ & $0^{c}$ & $0.01 \pm 0.00$ \\
\hline & Paracoccus & $0.28 \pm 0.24$ & $0.003 \pm 0.00^{\mathrm{a}}$ & $0.10 \pm 0.09$ \\
\hline & Pseudomonas & $1.51 \pm 0.95$ & $0.02 \pm 0.01^{\mathrm{a}}$ & $0.12 \pm 0.05^{\mathrm{b}}$ \\
\hline & U. Enterobacteriaceae & $2.40 \pm 2.05$ & $0.01 \pm 0.01^{\mathrm{a}}$ & $0.02 \pm 0.01^{\mathrm{b}}$ \\
\hline & U. Pseudomonadaceae & $0.73 \pm 0.34$ & $0.01 \pm 0.00^{\mathrm{a}}$ & $0.16 \pm 0.01$ \\
\hline & U. Sphingomonadaceae & $0.01 \pm 0.00$ & $0.001 \pm 0.00^{\mathrm{a}}$ & $0.01 \pm 0.01$ \\
\hline & U. Xanthomonadaceae & $0.70 \pm 0.27$ & $0.01 \pm 0.01^{\mathrm{a}}$ & $0.10 \pm 0.04$ \\
\hline Spirochaetes & Sphaerochaeta & 0 & $0.01 \pm 0.01^{\mathrm{a}}$ & 0 \\
\hline Tenericutes & ML615J_28 & $0.004 \pm 0.00$ & $0.01 \pm 0.00^{c}$ & 0 \\
\hline Verrucomicrobia & Akkermansia & $1.72 \pm 1.71$ & 0 & $0^{\mathrm{b}}$ \\
\hline
\end{tabular}

Relative abundance and taxonomic classification of key genera significantly different between PCS and CTRL (indicated by ${ }^{\mathrm{a}}$ ), PCnoS and CTRL (indicated by ${ }^{b}$ ), PCS and PCnoS (indicated by ${ }^{c}$ ), LEfSe algorithm analysis (LDA $>3$ with alpha<0.05) Data are mean \pm SEM. CTRL, healthy controls; PCS, smokers with periodontitis; PCnoS, non-smokers with periodontitis.

characterized by low abundance or absence of specific bacteria normally present in healthy subgingival microbiota. Low levels of Pedobacter, Granulicatella, Paracoccus, Gemellaceae, Clostridiaceae, Ruminococcaceae, Sphingomonadaceae, Pseudomonadaceae and of Xanthomondaceae and an unclassified genus of S24-7 were identified in PCS samples compared with CTRL, and low levels of Atopobium, Bifidobacterium, Coprococcus, Oridobacterium, Peptococcus, Oscillospira , Akkermansia and an unclassified genus of Moraxellaceae in PCnoS samples. These bacteria may, therefore, be relevant to the onset of periodontitis. To assess how an alteration of subgingival microbiota could impact periodontal disease, metagenomic function prediction was performed by PICRUSt analysis. Notably, genes associated with tissue damage were increased in PCS patients, while genes associated with amino acid metabolism and nutrient biosynthesis were reduced in PCS patients compared with CTRLs.

Although the PCS and PCnoS groups both exhibited a shift in subgingival bacterial composition compared with healthy subjects, several results highlight a major alteration in PCS 
A

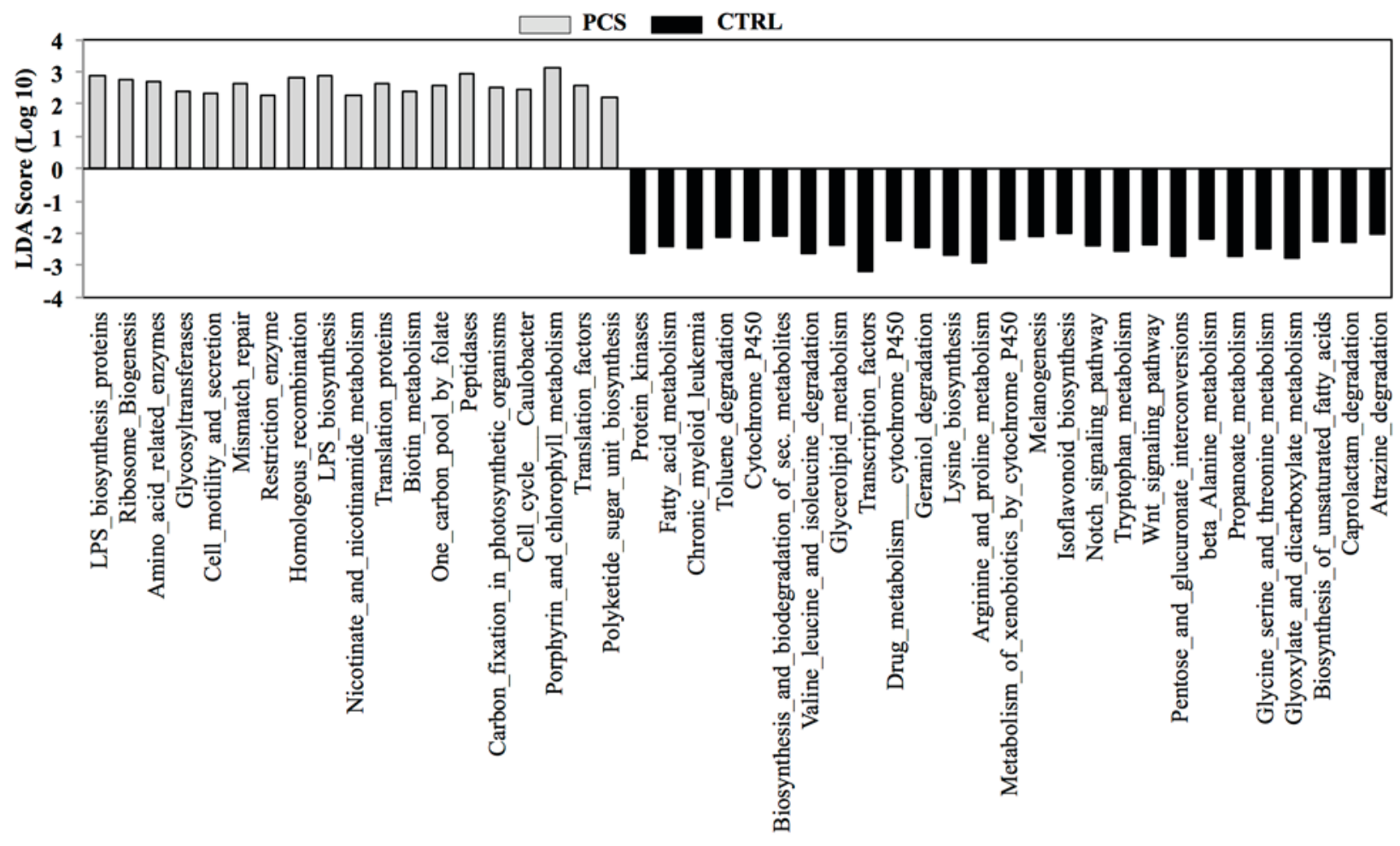

B

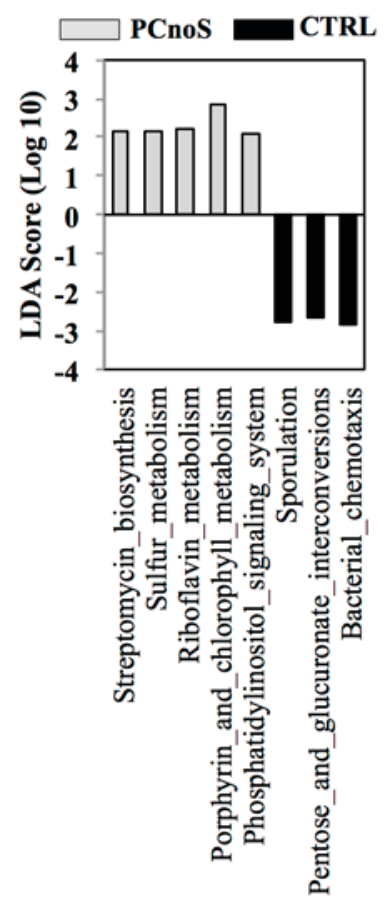

C

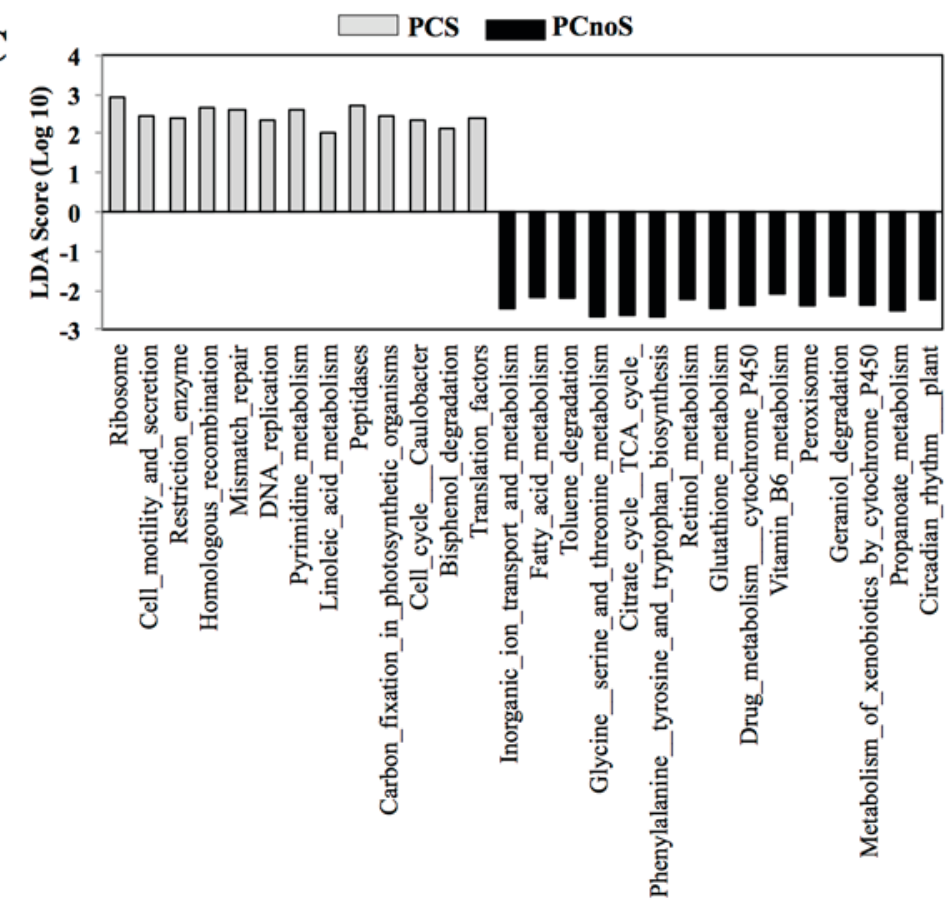

Figure 3. Predicted functional changes in the subgingival microbiome. Significantly different gene functions between (A) CTRL and PCS, (B) CTRL and PCnoS and (C) PCS and PCnoS groups. Differentially abundant gene functions derived from level 3 of the Kyoto Encyclopedia of Genes and Genomes database, were predicted using PICRUSt, and statistically significant pathways were identified using LDA effect size analysis (LDA score >2). PCS, smokers with periodontitis; CTRL, healthy controls; LDA, linear discriminative analysis.

smoker patients. In particular, the PCS subgingival microbiota was dominated by the Fusobacteria phylum, consistent with a lower bacterial richness. In addition, PCS samples displayed significant alterations in the relative abundance of several phyla compared to $\mathrm{PCnoS}$, and a specific bacterial signature discriminated PCS from CTRLs at the genus level. According to these results, predictive functional analysis designated PCS subgingival microbiota as potentially able to promote tissue damage, more so than the PCnoS microbiota.

Although the present study reports a detailed description of periodontitis-associated subgingival microbiota, further studies, involving larger cohorts and considering potential gender differences, would enhance our knowledge about the role of subgingival dysbiosis and smoking in periodontal 
disease. The present study indicated that chronic periodontitis results in a global perturbation of oral microbial communities, and elucidated the role of key bacteria in the complex ecology of subgingival microbiota. Finally, the present study confirmed that smoking aggravates the polymicrobial synergy of pathogenic bacteria and the subgingival dysbiosis that characterize chronic periodontal disease.

\section{Acknowledgements}

This work was partially funded by Regione Campania (2007 legge 5, to LoCh).

\section{References}

1. Avila M, Ojcius DM and Yilmaz O: The oral microbiota: Living with a permanent guest. DNA Cell Biol 28: 405-411, 2009.

2. Cho I and Blaser MJ: The human microbiome: At the interface of health and disease. Nat Rev Genet 13: 260-270, 2012.

3. Haffajee AD, Teles RP and Socransky SS: The effect of periodontal therapy on the composition of the subgingival microbiota. Periodontol 2000 42: 219-258, 2006.

4. Dentino A, Lee S, Mailhot J and Hefti AF: Principles of periodontology. Periodontol 2000 61: 16-53, 2013.

5. Al Harthi LS, Cullinan MP, Leichter JW and Thomson WM: The impact of periodontitis on oral health-related quality of life: A review of the evidence from observational studies. Aust Dent J 58: 274-277; quiz 384, 2013.

6. Seymour GJ, Ford PJ, Cullinan MP, Leishman S and Yamazaki K: Relationship between periodontal infections and systemic disease. Clin Microbiol Infect 13 (Suppl 4): S3-S10, 2007.

7. Armitage CG: Comparison of the microbiological features of chronic and aggressive periodontitis. Periodontol 200053 70-88, 2010.

8. Socransky SS, Haffajee AD, Cugini MA, Smith C and Kent RL Jr: Microbial complexes in subgingival plaque. J Clin Periodontol 25: 134-44, 1998.

9. Page RC and Kornman KS: The pathogenesis of human periodontitis: An introduction. Periodontol 2000 14: 9-11, 1997.

10. Lee J, Taneja V and Vassallo R: Cigarette smoking and inflammation: Cellular and molecular mechanisms. J Dent Res 91: 142-149, 2012.

11. Labriola A, Needleman I and Moles DR: Systematic review of the effect of smoking on nonsurgical periodontal therapy. Periodontol 2000 37: 124-137, 2005.

12. Haffajee AD and Socransky SS: Relationship of cigarette smoking to the subgingival microbiota. J Clin Periodontol 28 377-88, 2001.

13. Preber H, Bergström J and Linder LE: Occurrence of periopathogens in smoker and non-smoker patients. J Clin Periodontol 19: 667-671, 1992

14. Van der Velden U, Varoufaki A, Hutter JW, Xu L, Timmerman MF, Van Winkelhoff AJ and Loos BG: Effect of smoking and periodontal treatment on the subgingival microflora. J Clin Periodontol 30: 603-610, 2003.

15. Dewhirst FE, Chen T, Izard J, Paster BJ, Tanner AC, Yu WH, Lakshmanan A and Wade WG: The human oral microbiome. J Bacteriol 192: 5002-5017, 2010.

16. Sammartino G, Tia M, Gentile E, Marenzi G and Claudio PP: Platelet-rich plasma and resorbable membrane for prevention of periodontal defects after deeply impacted lower third molar extraction. J Oral Maxillofac Surg 67: 2369-2373, 2009.

17. Ramfjord SP: Present status of the modified Widman flap procedure. J Periodontol 48: 558-565, 1977.
18. Illumina. 16S Metagenomic Sequencing Library Preparation. 2013. Available: http://support.illumina.com/downloads/16s metagenomic sequencing library preparation.html

19. Mizrahi-Man O, Davenport ER and Gilad Y: Taxonomic classification of bacterial $16 \mathrm{~S}$ rRNA genes using short sequencing reads: Evaluation of effective study designs. PLoS One 8: e53608, 2013.

20. Illumina. MiSeq System Denature and Dilute Libraries Guide. 2016. Available:http://support.illumina.com/downloads/prepare libraries_for_sequencing_miseq_15039740.html

21. Zhang J, Kobert K, Flouri T and Stamatakis A: PEAR: A fast and accurate Illumina Paired-End reAd mergeR. Bioinformatics 30: 614-620, 2014.

22. Schmieder R and Edwards R: Quality control and preprocessing of metagenomic datasets. Bioinformatics 27: 863-864, 2011.

23. Caporaso JG, Kuczynski J, Stombaugh J, Bittinger K, Bushman FD, Costello EK, Fierer N, Peña AG, Goodrich JK, Gordon JI, et al: QIIME allows analysis of high-throughput community sequencing data. Nat Methods 7: 335-336, 2010.

24. DeSantis TZ, Hugenholtz P, Larsen N, Rojas M, Brodie EL, Keller K, Huber T, Dalevi D, Hu P and Andersen GL: Greengenes, a chimera-checked 16S rRNA gene database and workbench compatible with ARB. Appl Environ Microbiol 72: 5069-5072, 2006.

25. Clarke KR: Non-parametric multivariate analyses of changes in community structure. Aust J Ecol 18: 117-143, 1993.

26. Segata N, Izard J, Waldron L, Gevers D, Miropolsky L, Garrett WS and Huttenhower C: Metagenomic biomarker discovery and explanation. Genome Biol 12: R60, 2011.

27. Langille MG, Zaneveld J, Caporaso JG, McDonald D, Knights D, Reyes J, Clemente JC, Burkepile DE, Vega Thurber RL, Knight R, et al: Predictive functional profiling of microbial communities using 16S rRNA marker gene sequences. Nat Biotechnol 31: 814-821, 2013.

28. Kanehisa M and Goto S: KEGG: kyoto encyclopedia of genes and genomes. Nucleic Acids Res 28: 27-30, 2000.

29. Kim M, Morrison M and Yu Z: Evaluation of different partial 16S rRNA gene sequence regions for phylogenetic analysis of microbiomes. J Microbiol Methods 84: 81-87, 2011.

30. Hug LA, Baker BJ, Anantharaman K, Brown CT, Probst AJ, Castelle CJ, Butterfield CN, Hernsdorf AW, Amano Y, Ise K, et al: A new view of the tree of life. Nat Microbiol 1: 16048, 2016.

31. Wang J, Qi J, Zhao H, He S, Zhang Y, Wei S and Zhao F: Metagenomic sequencing reveals microbiota and its functional potential associated with periodontal disease. Sci Rep 3: 1843, 2013.

32. Kirst ME, Li EC, Alfant B, Chi YY, Walker C, Magnusson I and Wang GP: Dysbiosis and alterations in predicted functions of the subgingival microbiome in chronic periodontitis. Appl Environ Microbiol 81: 783-793, 2015.

33. Arora N, Mishra A and Chugh S: Microbial role in periodontitis: Have we reached the top? Some unsung bacteria other than red complex. J Indian Soc Periodontol 18: 9-13, 2014.

34. Bizzarro S, Loos BG, Laine ML, Crielaard W and Zaura E: Subgingival microbiome in smokers and non-smokers in periodontitis: An exploratory study using traditional targeted techniques and a next-generation sequencing. J Clin Periodontol 40: 483-492, 2013.

35. Colombo AP, Boches SK, Cotton SL, Goodson JM, Kent R, Haffajee AD, Socransky SS, Hasturk H, Van Dyke TE, Dewhirst $F$ and Paster BJ: Comparisons of subgingival microbial profiles of refractory periodontitis, severe periodontitis, and periodontal health using the human oral microbe identification microarray. J Periodontol 80: 1421-1432, 2009.

36. Gupta RS, Mahmood S and Adeolu M: Erratum: A phylogenomic and molecular signature based approach for characterization of the Phylum Spirochaetes and its major clades: Proposal for a taxonomic revision of the phylum. Front Microbiol 4: 322, 2013.

37. Bergeron MG, Simard P and Provencher P: Influence of growth medium and supplement on growth of Haemophilus influenzae and on antibacterial activity of several antibiotics. J Clin Microbiol 25: 650-655, 1987. 